\title{
Acute adrenal insufficiency following arthroplasty: a case report and review of the literature
}

\author{
Stylianos Mandanas, Maria Boudina, Alexandra Chrisoulidou, Katerina Xinou, Efterpi Margaritidou, \\ Spyros Gerou and Kalliopi Pazaitou-Panayiotou*
}

\begin{abstract}
Background: Acute adrenal insufficiency is a potentially lethal condition rarely caused by bilateral adrenal haemorrhage due to heparin use. Most of the times, it is difficult to establish the diagnosis, as symptoms are not specific. Few cases have been reported in the literature.

Case presentation: A 52-year-old Caucasian woman presented with abdominal pain, vomiting and weakness nine days after arthroplasty and heparin use. Hyperkalemia, low cortisol and high adrenocorticotropic hormone levels were found, indicating adrenal insufficiency. Magnetic resonance imaging of the upper abdomen was compatible with preceding adrenal haemorrhage. Hydrocortisone and fludrocortisone were administered. Review of the literature revealed 36 cases of postoperative adrenal haemorrhage which are presented briefly.

Conclusion: Postoperative acute adrenal insufficiency due to haemorrhage is a rare condition. If patients are treated based on clinical suspicion, they have good chances to survive. Hydrocortisone is given permanently in the majority of the patients.
\end{abstract}

Keywords: Adrenal insufficiency, Arthroplasty, Heparin

\section{Background}

Acute adrenal failure (AAF) is a potentially life-threatening complication presenting with non-specific symptoms as abdominal pain, nausea, fever, tachycardia, hypotension and lethargy [1]. However, hyponatremia and hyperkalemia as indicators of adrenal insufficiency should be evaluated very thoroughly [2].

AAF may occur in patients with previously undiagnosed primary adrenal insufficiency and sometimes after bilateral adrenal infarction or postoperative haemorrhage in otherwise healthy individuals [1]. The postoperative period carries a high risk for haemorrhage, as platelet consumption may lead to bleeding in vital organs, independently of anticoagulant administration. Additionally, the use of heparin represents an independent risk factor that predisposes to haemorrhage [3]. Injuries during procedures such as extracorporeal shock-wave lithotripsy or electroconvulsive therapy $[4,5]$ or use of certain materials and techniques may also predispose to coagulopathy and haemorrhage [6].

\footnotetext{
* Correspondence: kpazaitoupanayiotou@gmail.com

Department of Endocrinology, Theagenion Cancer Hospital, Al. Simeonidi st, Thessaloniki 54007, Greece
}

Moreover, hypothermia itself may play an important role in bleeding [6]. As signs and symptoms are not specific, they may easily lead to wrong diagnosis, such as postoperative septic shock or inflammation.

Total hip arthroplasty is a common surgical procedure associated with deep venous thrombosis and pulmonary embolism that are prevented by the use of anticoagulants $[7,8]$. The extensive use of anticoagulants in these operations explains the fact that bilateral adrenal haemorrhage is more often observed after knee or hip arthroplasty. However, orthopaedic surgery is probably associated with risk factors (other than anticoagulants) which lead to haemorrhage [9].

The use of low molecular weight heparin (LMWH) to avoid postoperative thromboembolic events can induce thrombocytopenia (heparin-induced thrombocytopenia [HIT]) and may lead to bilateral adrenal haemorrhage [2]. A pre-test clinical score (including thrombocytopenia, timing of platelet count fall, thrombosis, presence of other causes for thrombocytopenia, the so-called 4 T's) has been developed to establish the clinical suspicion of HIT [10], while heparin-platelet factor 4-immunoglobulin G (IgG)

\section{Biomed Central}

(c) 2013 Mandanas et al.; licensee BioMed Central Ltd. This is an Open Access article distributed under the terms of the Creative Commons Attribution License (http://creativecommons.org/licenses/by/2.0), which permits unrestricted use, distribution, and reproduction in any medium, provided the original work is properly cited. 
(PF4-IgG) antibodies and 14C-serotonin release assay are useful diagnostic tests [2].

The incidence of bilateral adrenal haemorrhage is estimated to be around 4.7-6.2 cases per million habitants in developed countries, but the prevalence is much higher in hospitalized patients, arising to $1.1 \%$ of them $[11,12]$. There are difficulties in establishing an early diagnosis of the disease [13] and adrenal haemorrhage is usually a post-mortem finding during autopsy performed to unravel the cause of death.

In addition to the elusive clinical presentation, imaging may also be deceiving: the enlarged haemorrhagic adrenals may be misdiagnosed as neoplastic masses, with irregular margins, though maintain their adreniform shape. Acute and subacute adrenal haemorrhage show high attenuation (50-90 HU) at unenhanced computed tomography (CT), without enhancement following intravenous (IV) contrast. In doubtful cases, decreased density and size of the adrenals during follow-up as well as the presence of calcifications may be extremely useful to confirm the diagnosis. Magnetic resonance imaging (MRI) is the most sensitive and specific imaging modality to confirm adrenal haemorrhage. Like $\mathrm{CT}$, the appearance of adrenal haemorrhage on MRI also depends on the progression of the bleeding. The most characteristic sign on MRI is the low signal ring on T2 sequences during the chronic phase [14].

The aim of the present work was a) to present a case of acute adrenal insufficiency caused by bilateral adrenal haemorrhage observed after arthroplasty and b) to summarize the published data regarding this rare and interesting clinical entity.

Computerized literature search was performed in the PubMed electronic database. The original query provided 71 possibly relevant articles. Furthermore, 10 articles were retrieved after searching the "Related Articles" link and the references. Of these, 34 were finally selected (including 36 case reports), whereas the remaining 47 articles were excluded for the following reasons: spontaneous bilateral adrenal haemorrhage without apparent cause $(n=9)$; concurrent diseases $(n=7)$; bilateral adrenal haemorrhage due to heparin for other reason, not postoperatively $(n=27)$; article in Japanese $(n=1)$; reviews and letters to the editor $(n=3)$.

In summary, 36 cases of postoperative bilateral adrenal haemorrhage have been documented. Mean age of patients during haemorrhage was 65.2 years (range 44-83) and there was no particular sex distribution. Abdominal pain, fever, vomiting and hypotension were the main symptoms at presentation, usually occurring between first and second week after surgery. Hyponatremia and hyperkalemia were the most common laboratory findings. In 27 out of 36 patients, diagnosis was made by CT scan, in two by abdominal ultrasound and in one by exploratory laparotomy. In four patients the diagnosis was confirmed after their death during autopsy and in two cases the imaging was not described. Nine patients succumbed to adrenal insufficiency.

Patients' clinical characteristics and diagnostic methods are summarized in Tables 1 and 2. Reported cases are divided into two groups: the first includes 21 patients with adrenal insufficiency after orthopaedic surgery (Table 1) and the second reports 15 patients with adrenal insufficiency after any other surgery (Table 2).

\section{Case presentation}

\section{Patients' history}

A 52-year-old Caucasian woman, mother of two children, underwent right hip arthroplasty and was administered LMWH (enoxaparin 8,000 IU per day) for seven days in order to prevent thromboembolic events. Arthroplasty was successfully completed without intraoperative or early postoperative complications, except for a fall in platelet count from $214,000 / \mu \mathrm{L}$ to $116,000 / \mu \mathrm{L}$. Haemoglobin and white blood cell count were normal. The patient was discharged from the hospital in good condition without any sign or symptom of haemorrhage, thromboembolism or infection.

\section{9th postoperative day, emergency department}

On postoperative day 9, she presented in the emergency department complaining of abdominal pain, vomiting and weakness. She was dehydrated and tachycardic (105 beats per minute). Decreased skin turgor and low blood pressure $(90 / 60 \mathrm{~mm} \mathrm{Hg})$ were observed. Biochemical exams indicated hyponatremia $(128 \mathrm{mmol} / \mathrm{L}$, normal range 136-145) and hyperkalemia $(5.97 \mathrm{mmol} / \mathrm{L}$, normal range 3.5-5.1), normal serum glucose levels, as well as normal kidney and liver function. The patient was afebrile and the wound healed satisfactory. The administration of isotonic solutions was decided but the patient responded poorly. Intravenous use of dopamine was added thereafter, resulting in slight improvement of clinical symptoms, mainly vomiting and arterial blood pressure. As the abdominal pain was persistent, the patient underwent abdominal CT which was indicative of "bilateral adrenal adenomas". Due to this finding, the patient was referred to our department for further evaluation.

\section{Referral to the endocrinology department}

Considering the clinical signs of dehydration and the presence of hyperkalemia $(5.72 \mathrm{mmol} / \mathrm{L}$, normal range $3.5-5.1)$ and hyponatremia $(130 \mathrm{mmol} / \mathrm{L}$, normal range 136-145), adrenal insufficiency was suspected. Cortisol levels were measured and found to be very low (cortisol 40nmol/l, normal range $70-250 \mathrm{nmol} / \mathrm{l})$. A $250-\mu \mathrm{g}$ adrenocorticotropic hormone $(\mathrm{ACTH})$ stimulation test (Synacthen test) was performed for further evaluation; no increase in cortisol was observed confirming the diagnosis of primary adrenal 
Table 1 Adrenal insufficiency after orthopaedic surgery

\begin{tabular}{|c|c|c|c|c|c|c|c|c|}
\hline Cases & Author & Country & Age & Sex & Type of surgery & Diagnosis & Symptoms & Biochemical exams \\
\hline 1 & $\begin{array}{l}\text { Findling et al., } \\
1987[15]\end{array}$ & USA & 44 & M & unspecified & $C T$ & $\begin{array}{l}\text { abdominal pain, } \\
\text { vomiting, hypotension }\end{array}$ & $\begin{array}{l}\text { hyponatremia, } \\
\text { hyperkalemia }\end{array}$ \\
\hline 2 & $\begin{array}{l}\text { Delhumeau et al., } \\
1989 \text { [16] }\end{array}$ & France & 76 & $\mathrm{~F}$ & total hip arthroplasty & $\begin{array}{l}\text { abdominal } \\
\text { ultrasound }\end{array}$ & $\begin{array}{l}\text { abdominal pain, fever, } \\
\text { hypotension, altered } \\
\text { consciousness }\end{array}$ & $\begin{array}{l}\text { hyponatremia, } \\
\text { natriuresis, } \\
\text { thrombocytopenia }\end{array}$ \\
\hline 3 & & & 74 & M & tibial osteosynthesis & not done & $\begin{array}{l}\text { abdominal pain, fever, } \\
\text { hypotension, shock, } \\
\text { altered consciousness }\end{array}$ & $\begin{array}{l}\text { hyponatremia, } \\
\text { thrombocytopenia }\end{array}$ \\
\hline 4 & & & 62 & M & tibial osteosynthesis & $C T$ & $\begin{array}{l}\text { abdominal pain, } \\
\text { asthenia, nausea }\end{array}$ & $\begin{array}{l}\text { hyponatremia, } \\
\text { thrombocytopenia }\end{array}$ \\
\hline 5 & $\begin{array}{l}\text { Ernest and Fischer, } \\
1991[17]\end{array}$ & Australia & 68 & $\mathrm{~F}$ & total hip arthroplasty & $C T$ & fever, hypotension & $\begin{array}{l}\text { hyponatremia, } \\
\text { hyperkalemia }\end{array}$ \\
\hline 6 & $\begin{array}{l}\text { Souied et al., } \\
1991 \text { [18] }\end{array}$ & France & 63 & $\mathrm{~F}$ & total hip arthroplasty & $C T$ & fever, hypotension & $\begin{array}{l}\text { hyponatremia, } \\
\text { hyperkalemia }\end{array}$ \\
\hline 7 & $\begin{array}{l}\text { Bleasel et al., } \\
1992[19]\end{array}$ & Australia & 69 & $\mathrm{~F}$ & total knee arthroplasty & $C T$ & $\begin{array}{l}\text { fever, nausea, vomiting, } \\
\text { abdominal pain, hypotension }\end{array}$ & $\begin{array}{l}\text { hyponatremia, } \\
\text { hyperkalemia }\end{array}$ \\
\hline 8 & $\begin{array}{l}\text { Hardwicke and } \\
\text { Kisly, } 1992 \text { [20] }\end{array}$ & USA & 63 & $\mathrm{~F}$ & $\begin{array}{l}\text { bilateral total knee } \\
\text { arthroplasty }\end{array}$ & $C T$ & $\begin{array}{l}\text { fever, nausea, anorexia, } \\
\text { vomiting, abdominal pain, } \\
\text { confusion, feeling of illness, } \\
\text { hypotension }\end{array}$ & $\begin{array}{l}\text { anemia, } \\
\text { hyponatremia, } \\
\text { hyperkalemia }\end{array}$ \\
\hline 9 & $\begin{array}{l}\text { Delhumeau et al., } \\
1993 \text { [21] }\end{array}$ & France & 74 & M & $\begin{array}{l}\text { total hip arthroplasty, } \\
\text { thrombectomy of both } \\
\text { limbs due to bilateral } \\
\text { arterial thrombosis }\end{array}$ & $C T$ & $\begin{array}{l}\text { abdominal pain, fever, } \\
\text { hypotension, abdominal } \\
\text { tenderness }\end{array}$ & $\begin{array}{l}\text { hyponatremia, } \\
\text { hyperkalemia }\end{array}$ \\
\hline 10 & $\begin{array}{l}\text { Santonastaso et al., } \\
1993 \text { [22] }\end{array}$ & Italy & $\begin{array}{l}\text { not } \\
\text { reported }\end{array}$ & $\mathrm{F}$ & osteotomy & $C T$ & $\begin{array}{l}\text { somnolence, asthenia, } \\
\text { hypotension }\end{array}$ & not reported \\
\hline 11 & $\begin{array}{l}\text { Ries et al., } \\
1994 \text { [23] }\end{array}$ & USA & 61 & $\mathrm{~F}$ & $\begin{array}{l}\text { bilateral total knee } \\
\text { arthroplasty }\end{array}$ & autopsy & $\begin{array}{l}\text { abdominal discomfort, } \\
\text { nausea, collapse }\end{array}$ & none \\
\hline 12 & $\begin{array}{l}\text { Cozzolino et al., } \\
1997 \text { [24] }\end{array}$ & USA & 66 & $\mathrm{~F}$ & total knee arthroplasty & $C T$ & $\begin{array}{l}\text { nausea, anorexia, } \\
\text { emesis, lethargy }\end{array}$ & $\begin{array}{l}\text { hyponatremia, } \\
\text { hyperkalemia, } \\
\text { anemia, azotemia }\end{array}$ \\
\hline 13 & $\begin{array}{l}\text { Rowland et al., } \\
1999[25]\end{array}$ & Australia & 50 & M & unspecified & $C T$ & $\begin{array}{l}\text { fever, abdominal pain, } \\
\text { dizziness, hypotension }\end{array}$ & hyponatremia \\
\hline 14 & $\begin{array}{l}\text { Caubet et al., } \\
1999[26]\end{array}$ & France & 63 & $\mathrm{~F}$ & tibial osteosynthesis & $C T$ & $\begin{array}{l}\text { fever, hypotension, } \\
\text { tachypnea }\end{array}$ & $\begin{array}{l}\text { thrombocytopenia, } \\
\text { hyponatremia, } \\
\text { hyperkalemia, } \\
\text { elevated INR }\end{array}$ \\
\hline 15 & $\begin{array}{l}\text { Scheffold et al., } \\
2001[27]\end{array}$ & Germany & 63 & M & $\begin{array}{l}\text { intracondylar nail- } \\
\text { extension }\end{array}$ & $\begin{array}{l}\text { abdominal } \\
\text { ultrasound }\end{array}$ & abdominal pain, fever & leukocytosis \\
\hline 16 & $\begin{array}{l}\text { LaBan et al., } \\
2003 \text { [28] }\end{array}$ & USA & 82 & $\mathrm{~F}$ & $\begin{array}{l}\text { bilateral knee } \\
\text { arthroplasty }\end{array}$ & $C T$ & abdominal pain, nausea & $\begin{array}{l}\text { elevated INR, } \\
\text { hyponatremia }\end{array}$ \\
\hline 17 & $\begin{array}{l}\text { Schuchmann et al., } \\
2005 \text { [8] }\end{array}$ & USA & 83 & $\mathrm{~F}$ & $\begin{array}{l}\text { bilateral total knee } \\
\text { arthroplasty }\end{array}$ & autopsy & $\begin{array}{l}\text { anxiety, hypertension, } \\
\text { midback pain, shortness } \\
\text { of breath, shock }\end{array}$ & $\begin{array}{l}\text { hyponatremia, } \\
\text { hypochloremia, } \\
\text { leukocytosis, anemia, } \\
\text { elevated INR }\end{array}$ \\
\hline 18 & $\begin{array}{l}\text { Kurtz and Yang, } \\
2007[29]\end{array}$ & USA & 54 & $\mathrm{~F}$ & total hip arthroplasty & $C T$ & $\begin{array}{l}\text { fever, abdominal pain, } \\
\text { orthostatic syncope }\end{array}$ & not reported \\
\hline 19 & $\begin{array}{l}\text { Mongardon et al., } \\
2007 \text { [30] }\end{array}$ & France & 64 & M & total hip arthroplasty & $C T$ & $\begin{array}{l}\text { fever, abdominal pain, } \\
\text { confusion, hypotension }\end{array}$ & $\begin{array}{l}\text { thrombocytopenia, } \\
\text { high urinary sodium }\end{array}$ \\
\hline 20 & $\begin{array}{l}\text { Thota et al., } \\
2012 \text { [31] }\end{array}$ & USA & 68 & M & $\begin{array}{l}\text { bilateral total knee } \\
\text { arthroplasty }\end{array}$ & $C T$ & $\begin{array}{l}\text { fever, abdominal discomfort, } \\
\text { anorexia, fatigue, hypertension, } \\
\text { tachycardia, altered mental status }\end{array}$ & $\begin{array}{l}\text { hyperglycemia, } \\
\text { leukocytosis, anemia, } \\
\text { thrombocytopenia, } \\
\text { hyponatremia }\end{array}$ \\
\hline 21 & $\begin{array}{l}\text { Chow et al., } \\
2012 \text { [32] }\end{array}$ & USA & 44 & M & $\begin{array}{l}\text { bilateral total knee } \\
\text { arthroplasty }\end{array}$ & $C T$ & $\begin{array}{l}\text { hypotension, abdominal } \\
\text { pain, tachycardia, fever }\end{array}$ & $\begin{array}{l}\text { leukocytosis, } \\
\text { D-dimers elevation, } \\
\text { hyponatremia, } \\
\text { thrombocytopenia }\end{array}$ \\
\hline
\end{tabular}


Table 2 Adrenal insufficiency after any surgery except for orthopaedic surgery

\begin{tabular}{|c|c|c|c|c|c|c|c|c|}
\hline Cases & Author & Country & Age & Sex & Type of surgery & Diagnosis & Symptoms & Lab results \\
\hline 1 & Steer and Fromm, 1980 [33] & USA & 71 & M & cholecystectomy & not done & $\begin{array}{l}\text { abdominal pain, fever, vomiting, } \\
\text { anorexia, weakness, hypotension }\end{array}$ & $\begin{array}{l}\text { eosinophilia, hyponatremia, } \\
\text { hyperkalemia, prothrombin } \\
\text { time elevation }\end{array}$ \\
\hline 2 & Jacobson et al., 1986 [34] & USA & 55 & M & retropubic prostatectomy & $C T$ & $\begin{array}{l}\text { fever, tachycardia, hypotension, } \\
\text { dyspnea, abdominal pain, nausea, } \\
\text { vomiting, diarrhea, ileus }\end{array}$ & hyponatremia, hyperkalemia \\
\hline 3 & Miller et al., 1986 [35] & USA & 81 & $\mathrm{~F}$ & aorto-iliac aneurysmectomy & $C T$ & $\begin{array}{l}\text { fever, nausea, vomiting, abdominal } \\
\text { pain, lethargy }\end{array}$ & $\begin{array}{l}\text { hyponatremia, hyperkalemia, } \\
\text { leukocytosis }\end{array}$ \\
\hline 4 & Homcy and Southern, 1989 [36] & USA & 71 & M & colectomy & autopsy & confusion, hypotension & hyponatremia \\
\hline 5 & Ting et al., 1992 [37] & USA & 62 & M & coronary bypass grafting & $C T$ & $\begin{array}{l}\text { left flank pain, fever, tachypnea, } \\
\text { tachycardia, tired, hypotension }\end{array}$ & $\begin{array}{l}\text { leukocytosis, hyponatremia, } \\
\text { hyperkalemia }\end{array}$ \\
\hline 6 & Leschi et al., 1994 [38] & France & 63 & M & aortofemoral bypass graft & autopsy & confusion & hyponatremia, hyperkalemia \\
\hline 7 & Belmore and Walters, 1995 [39] & USA & 53 & M & laparoscopic cholecystectomy & $C T$ & $\begin{array}{l}\text { abdominal pain, anorexia, fatigue, } \\
\text { hypotension, dehydration }\end{array}$ & $\begin{array}{l}\text { hyponatremia, hyperkalemia, } \\
\text { hyperamylasemia, partial } \\
\text { thromboplastin time prolonged }\end{array}$ \\
\hline 8 & Scheiwiller et al., 2002 [40] & Switzerland & 71 & M & low anterior rectum resection & $\mathrm{CT}$ & $\begin{array}{l}\text { anorexia, abdominal pain, fever, } \\
\text { hypotension }\end{array}$ & hyponatremia \\
\hline 9 & Sousa Escandon et al., 2002 [41] & Spain & 82 & M & $\begin{array}{l}\text { partial nephrectomy for } \\
\text { renal adenocarcinoma }\end{array}$ & $C T$ & $\begin{array}{l}\text { abdominal pain, nausea, fever, } \\
\text { hypotension, severe respiratory distress }\end{array}$ & $\begin{array}{l}\text { hyponatremia, azotemia, anemia, } \\
\text { leukocytosis, thrombocytosis }\end{array}$ \\
\hline 10 & Bakaeen et al., 2005 [42] & USA & 51 & M & coronary artery bypass graft & $C T$ & $\begin{array}{l}\text { fever, abdominal pain, diarrhea, } \\
\text { orthostatic hypotension, tachycardia }\end{array}$ & thrombocytopenia \\
\hline 11 & Gutenberg et al., 2007 [43] & Germany & 69 & M & intracranial tumor surgery & exploratory laparotomy & hypotension, abdomen tense & not reported \\
\hline 12 & Munoz Corsini et al., 2008 [44] & Spain & 63 & M & right hemicolectomy & $\mathrm{CT}$ & $\begin{array}{l}\text { disorientation, difficulty in breathing, } \\
\text { tachycardia, fever }\end{array}$ & $\begin{array}{l}\text { leukocytosis, anemia, increase in } \\
\text { fibrinogen, }\end{array}$ \\
\hline 13 & Peel and Whitelaw, 2011 [45] & USA & 60 & M & right hemicolectomy & $C T$ & $\begin{array}{l}\text { backache, fever, hypotension, } \\
\text { agitation, confusion }\end{array}$ & $\begin{array}{l}\text { hyponatremia, thrombocytopenia, } \\
\text { leukocytosis }\end{array}$ \\
\hline 14 & Rosenberger et al., 2011 [2] & USA & 69 & M & $\begin{array}{l}\text { gastrectomy, esophagojejuno- } \\
\text { stomy, cholecystectomy }\end{array}$ & $C T$ & $\begin{array}{l}\text { abdominal and flank pain, } \\
\text { tachypnea, tachycardia, } \\
\text { hypotension, oliguria, } \\
\text { myocardial infarction }\end{array}$ & oliguric renal failure \\
\hline 15 & Balsach Sole et al., 2012 [46] & Spain & 70 & M & cephalic duodenopancreatectomy & $C T$ & lethargy, hypotension & hyponatremia, hypoglycemia \\
\hline
\end{tabular}



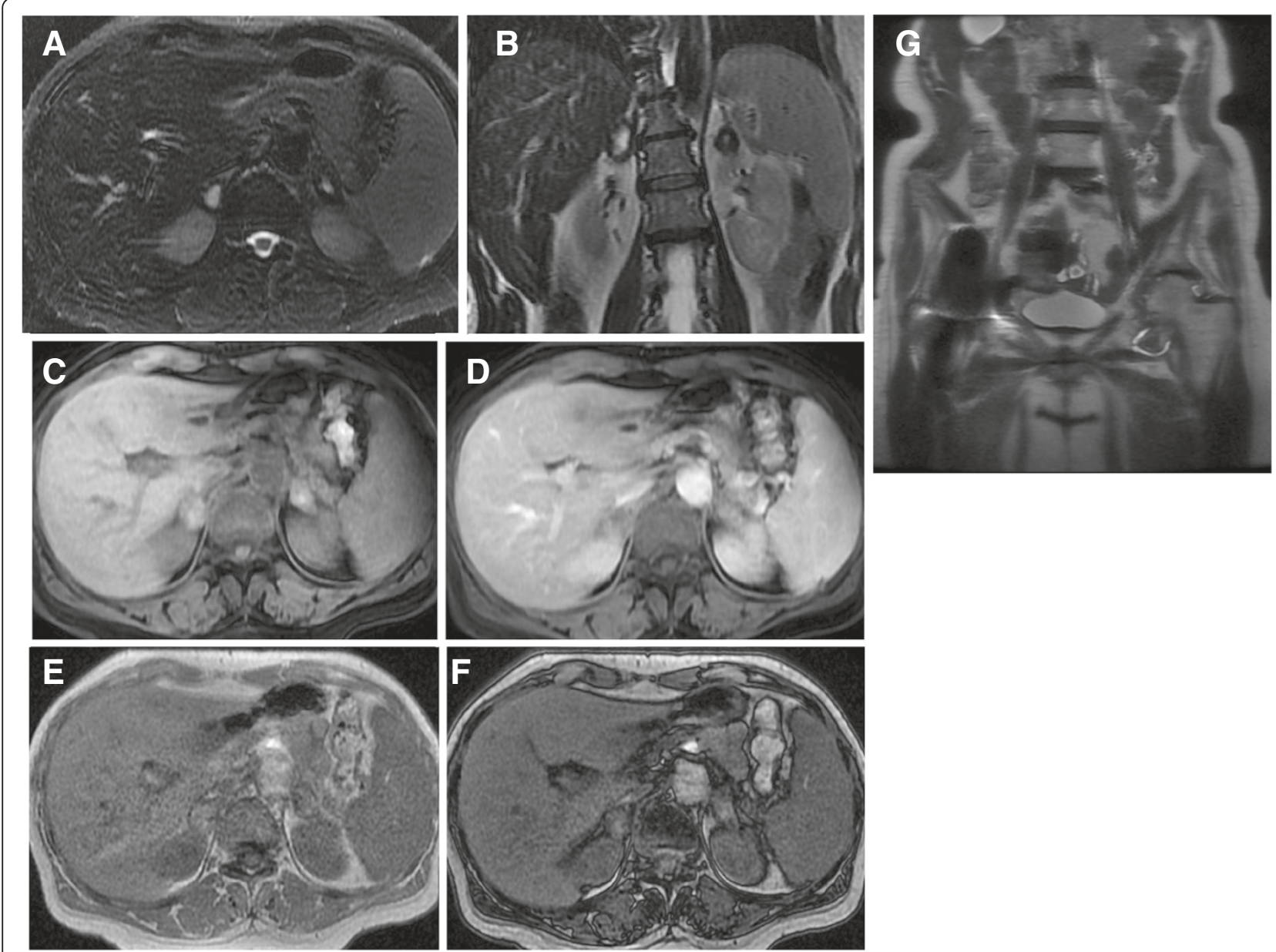

Figure 1 Abdominal magnetic resonance imaging obtained 15 days after surgery. Axial (A) T2 weighted image with fat suppression coronal (B) T2 weighted image, in and out of phase axial (C, D) T1 images, axial (E, F) T1 weighted images with fat saturation before and after contrast administration, coronal (G) T2-weighted image. Bilateral adrenal lesions showing high signal both on T1-weighted (E) and on T2-weighted images (A, B), and low signal rim on T2-weighted images (A, B), representing haemosiderin. The lesions do not show any signal drop-off on $\mathrm{T} 1 \mathrm{in}-\mathbf{( C )}$ and out- of phase images (D). After intravenous contrast administration (F) no enhancement is depicted. These findings are consistent with chronic adrenal hematomas. Note the signal void in the right pelvis due to the presence of a metallic right hip prosthesis.

insufficiency (basal cortisol equal to $32.9 \mathrm{nmol} / \mathrm{l}, 30$ and 60 min after Synacthen equal to $34 \mathrm{nmol} / 1$ and $32.1 \mathrm{nmol} / \mathrm{l}$ respectively, normal range $70-250 \mathrm{nmol} / \mathrm{l})$. ACTH levels were very high $(1763.4 \mathrm{pg} / \mathrm{ml}$, normal range 9-52). MRI of the upper abdomen showed the presence of bilateral adrenal "lesions" with greatest dimension $2.3 \mathrm{~cm}$ on the right and $2.5 \mathrm{~cm}$ on the left. On T2 weighted-images the above mentioned findings had high signal intensity with a low signal intensity ring along the periphery compatible with the presence of haemosiderin consequence of previous haemorrhage and chronic hematomas (Figure 1). No signal drop-off on in- and out- of phase images and no significant enhancement following iv contrast was depicted.

\section{Diagnostic evaluation and therapy}

Considering the variety of causes that could trigger bilateral adrenal haemorrhage in accordance with the medical history of the patient, traumatic injury, burns or pregnancy were ruled out [13]. Furthermore, she was afebrile without any clinical signs of septic shock. Antiphospholipid syndrome was excluded due to the absence of vascular thrombosis and the history of two normal deliveries. Based on the fact that the patient had received LMWH postoperatively, haemorrhage was considered to be the cause of adrenal failure. Heparin-PF4-IgG antibodies (measured by enzyme-linked immunosorbent assay [ELISA]) were negative. Moreover, the low pretest clinical score for HIT (total 2 points, 1 point from thrombocytopenia and 1 point from surgery) in the patient was correlated with high- negative predictive value for heparin induced adrenal haemorrhage [10].

Replacement therapy with hydrocortisone and fludrocortisone was started. Three months later, ACTH levels fell to $397 \mathrm{pg} / \mathrm{ml}$. Adrenal MRI showed that the lesions had decreased in size and had homogeneous low 

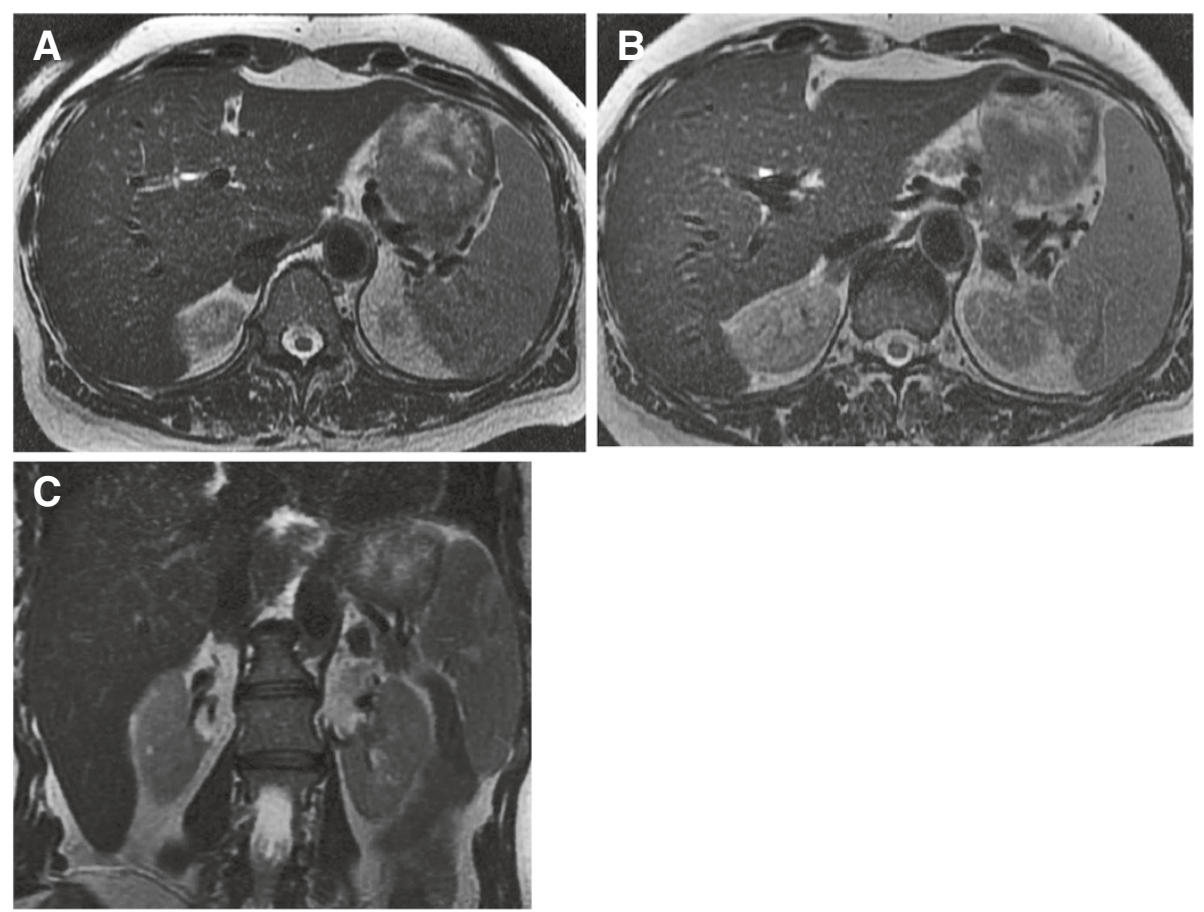

Figure 2 Abdominal magnetic resonance imaging obtained 3.5 months after surgery. Axial (A, B) and coronal (C) T2-weighted images showing a decrease in size and a change of signal of the bilateral adrenal hematomas. The lesions now have homogeneous low signal, due to haemosiderin.

signal on T2 weighted images, findings consistent with the evolution of hematomas (Figure 2). The oral replacement with hydrocortisone and fludrocortisone remains until the present time.

\section{Conclusions}

Bilateral adrenal haemorrhage is a rare disease which can follow major surgical operations. It should be suspected in patients presenting with fever, abdominal pain, confusion and hemodynamic collapse not responding to standard medical treatment [31]. The increased incidence after orthopaedic surgery, the association with anticoagulants use and the great mortality in misdiagnosed cases should keep physicians alerted.

\section{Consent}

Written informed consent was obtained from the patient for publication of this Case Report and any accompanying images. A copy of the written consent is available for review by the Editor-in-Chief of this journal.

\section{Abbreviations}

AAF: Acute adrenal failure; LMWH: Low molecular weight heparin; HIT: Heparin induced thrombocytopenia; IgG: Immunoglobulin G; PF4IgG: Platelet factor 4- immunoglobulin G; CT: Computed tomography; IV: Intravenous; MRI: Magnetic resonance imaging; ACTH: Adrenocorticotropic hormone; ELISA: Enzyme-linked immunosorbent assay.
Competing interests

The authors declare that they have no competing interests.

\section{Authors' contributions}

SM and MB reviewed the literature and prepared the draft. KX performed and discussed imaging. EM collected patients' clinical data. SG performed the immunoassay. AC and KPP conceived the idea and made the amendments of the manuscript. All authors read and approved the final manuscript.

\section{Disclosure statement}

The authors have nothing to disclose.

Received: 5 July 2013 Accepted: 9 September 2013

Published: 12 September 2013

\section{References}

1. Bouillon R: Acute adrenal insufficiency. Endocrinol Metab Clin North Am 2006, 35:767-775

2. Rosenberger LH, Smith PW, Sawyer RG, Hanks JB, Adams RB, Hedrick TL: Bilateral adrenal hemorrhage: the unrecognized cause of hemodynamic collapse associated with heparin-induced thrombocytopenia. Crit Care Med 2011, 39:833-838.

3. Chang JC: Review: Postoperative thrombocytopenia: with etiologic, diagnostic, and therapeutic consideration. Am J Med Sci 1996, 311:96-105.

4. Donald IP, Freeman CP: Adrenal hemorrhagic necrosis following electroconvulsive therapy. Lancet 1982, 31:277.

5. Lai YL, Chang WC, Huang HH: Obscure abdominal pain in a 55 -year-old man. Diagnosis: Intra-abdominal hemorrhage with adrenal hematoma. Gastroenterology 2010, 139:387.

6. McKenna R: Abnormal coagulation in the postoperative period contributing to excessive bleeding. Med Clin North Am 2001, 85:1277-1310 
7. Geerts WH: Prevention of venous thromboembolism: American College of Chest Physicians Evidence-Based Clinical Practice Guidelines (8th Edition). Chest 2008, 133(Suppl 6):381-453.

8. Schuchmann JA, Friedman PA: Bilateral adrenal hemorrhage: an unusual complication after bilateral total knee arthroplasty. Am J Phys Med Rehabil 2005, 84:899-903.

9. Oberweis BS, Nukala S, Rosenberg A, Guo Y, Stuchin S, Radford MJ, Berger JS: Thrombotic and bleeding complications after orthopedic surgery. Am Heart J 2013, 165:427-433.

10. Lo GK, Juhl D, Warkentin TE, Sigouin CS, Eichler P, Greinacher A: Evaluation of pretest clinical score (4 T's) for the diagnosis of heparin-induced thrombocytopenia in two clinical settings. J Thromb Haemost 2006, 4:759-765.

11. Bharucha T, Broderick C, Easom N, Roberts C, Moore D: Bilateral adrenal haemorrhage presenting as epigastric and back pain. JRSM Short Rep 2012, 3:15

12. Vella A, Nippoldt TB, Morris JC 3rd: Adrenal hemorrhage: a 25-year experience at the Mayo Clinic. Mayo Clin Proc 2001, 76:161-168.

13. Arlt W, Allolio B: Adrenal insufficiency. Lancet 2003, 361:1881-1893.

14. Wah TM, Guthrie JA, Joyce AD: Cross-Sectional Imaging of adrenal masses. In Imaging in Oncological Urology. Edited by De la Rosette JJMCH, Manyak MJ, Harisinghani MG, Wijkstra H. London: Springer; 2009:5-27.

15. Findling JW, Korducki JM, Lahiri PK, Miller DD, Raff H: Bilateral adrenal hemorrhage associated with heparin-induced thrombocytopenia. Wis Med J 1987, 86:27-29.

16. Delhumeau A, Houet JF, Bourrier P, Bukowski JG, Granry JC: Heparin-induced thrombocytopenia complicated by hematoma of the adrenal glands and acute adrenal insufficiency. Ann Fr Anesth Reanim 1989, 8:656-658.

17. Ernest D, Fisher MM: Heparin-induced thrombocytopaenia complicated by bilateral adrenal haemorrhage. Intensive Care Med 1991, 17:238-240

18. Souied F, Pourriat JL, Le Roux G, Hoang P, Kemeny JL, Cupa M: Adrenal hemorrhagic necrosis related to heparin-associated thrombocytopenia. Crit Care Med 1991, 19:297-299.

19. Bleasel JF, Rasko JE, Rickard KA, Richards G: Acute adrenal insufficiency secondary to heparin-induced thrombocytopenia-thrombosis syndrome. Med J Aust 1992, 157:192-193.

20. Hardwicke MB, Kisly A: Prophylactic subcutaneous heparin therapy as a cause of bilateral adrenal hemorrhage. Arch Intern Med 1992, 152:845-847.

21. Delhumeau A, Moreau X, Chapotte C, Houi N, Bigorgne JC: Heparin-associated thrombocytopenia syndrome: an underestimated etiology of adrenal hemorrhage. Intensive Care Med 1993, 19:475-477.

22. Santonastaso M, Bovo P, Colaceci R, Corbanese U, Ruga P: Acute adrenal failure due to adrenal hemorrhagic necrosis secondary to heparin-induced thrombocytopenia. Recenti Prog Med 1993, 84:687-690.

23. Ries MD, Guiney $\mathrm{W} J \mathrm{~J}$, Lynch F: Fatal massive adrenal hemorrhage after bilateral total knee arthroplasty. J Arthroplasty 1994, 9:559-562.

24. Cozzolino D, Peerzada J, Heaney JA: Adrenal insufficiency from bilatera adrenal hemorrhage after total knee replacement surgery. Urology 1997 , 50:125-127.

25. Rowland CH, Woodford PA, De Lisle-Hammond J, Nair B: Heparin-induced thrombocytopenia-thrombosis syndrome and bilateral adrenal haemorrhage after prophylactic heparin use. Aust N Z J Med 1999, 29:741-742

26. Caubet O, Pillet O, Cherifi A, Mayet T, Castaing Y, Favarel Garrigues JC: Acute adrenal insufficiency due to bilateral adrenal hematoma following severe thrombopenia induced by low-molecular-weight heparin. Presse Med 1999, 28:1010-1012.

27. Scheffold N, Schöngart H, Berentelg J, Prager P, Cyran J: Rare complication of a heparin-induced thrombocytopenia type II. Dtsch Med Wochenschr 2001, 126:329-333.

28. LaBan MM, Whitmore CE, Taylor RS: Bilateral adrenal hemorrhage after anticoagulation prophylaxis for bilateral knee arthroplasty. Am J Phys Med Rehabil 2003, 82:418-420.

29. Kurtz LE, Yang S: Bilateral adrenal hemorrhage associated with heparin induced thrombocytopenia. Am J Hematol 2007, 82:493-494

30. Mongardon N, Bruneel F, Henry Lagarrigue M, Legriel S, Revault D'Allonnes L, Guezennec P, Troche G, Bedos JP: Shock during heparin-induced thrombocytopenia: look for adrenal insufficiency. Intensive Care Med 2007, 33:547-548.
31. Thota R, Porter J, Ganti AK, Peters E: Hemodynamic collapse following bilateral knee arthroplasty: a mysterious case. J Thromb Thrombolysis 2012, 33:3-5.

32. Chow WW, Abnousi F, Huddleston Jl, Lin LH: Heparin-induced thrombocytopenia after total knee arthroplasty, with subsequent adrenal hemorrhage. J Arthroplasty 2012, 27:15-18. 1413.e.

33. Steer M, Fromm D: Recognition of adrenal insufficiency in the postoperative patient. Am J Surg 1980, 139:443-446.

34. Jacobson SA, Blute RD Jr, Green DF, McPhedran P, Weiss RM, Lytton B: Acute adrenal insufficiency as a complication of urological surgery. $J$ Urol 1986, 135:337-340

35. Miller EH, Woldenberg DH, Gittler RD, Zumoff B: Bilateral adrenal hemorrhage following surgery. N Y State J Med 1986, 86:651-653.

36. Homcy CJ, Southern JF: Case records of the Massachusetts General Hospital. Weekly clinicopathological exercises. Case 49-1989. A 71-year -old man with thrombocytopenia and hypotension after resection of a colonic carcinoma. N Engl J Med 1989, 321:1595-1603.

37. Ting W, Nosher JL, Scholz PM, Spotnitz AJ: Bilateral adrenal hemorrhage after an open heart operation. Ann Thorac Surg 1992, 54:357-358.

38. Leschi JP, Goëau-Brissonnière O, Coggia M, Chiche L: Heparin-related thrombocytopenia and adrenal hemorrhagic necrosis following aortic surgery. Ann Vasc Surg 1994, 8:506-508.

39. Belmore DJ, Walters DN: Bilateral adrenal hemorrhage following laparoscopic cholecystectomy. Surg Endosc 1995, 9:919-920.

40. Scheiwiller A, Morel P, Soravia C: Bilateral adrenal gland hemorrhage after anterior deep rectum resection. Case report with review of the literature. Chirurg 2002, 73:628-632

41. Sousa Escandón A, Mateos A, Sánchez F, González A, García R, Pérez J, Pulpeiro JR, Uribarri C: Massive bilateral adrenal hemorrhage after conservative tumor surgery in the isthmus of a horseshoe kidney. Actas Urol Esp 2002, 26:420-424.

42. Bakaeen FG, Walkes JC, Reardon MJ: Heparin-induced thrombocytopenia associated with bilateral adrenal hemorrhage after coronary artery bypass surgery. Ann Thorac Surg 2005, 79:1388-1390.

43. Gutenberg A, Lange B, Gunawan B, Larsen J, Brück W, Rohde V, Verheggen R: Spontaneous adrenal hemorrhage: a little-known complication of intracranial tumor surgery. Case report. J Neurosurg 2007, 106:1086-1088.

44. Munoz Corsini L, Delgado Arnaiz C, Garcia Del Valle S, Reboto Cortes P, Lopez Del Castillo A: Postoperative bilateral adrenal hemorrhage: correlation between clinical and radiological signs. J Clin Anesth 2008, 20:605-608.

45. Peel N, Whitelaw SC: Bilateral adrenal haemorrhage following right hemicolectomy. Int J Colorectal Dis 2011, 26:681-682.

46. Balsach Solé A, Oms Bernat LM, Garrido Romero M, Mato Ruiz R, Sala-Pedrós J: Bilateral adrenal haemorrhage after cephalic duodenopancreatectomy. Cir Esp 2012, 90:56-57.

doi:10.1186/1756-0500-6-370

Cite this article as: Mandanas et al.: Acute adrenal insufficiency

following arthroplasty: a case report and review of the literature. BMC Research Notes 2013 6:370.

\section{Submit your next manuscript to BioMed Central and take full advantage of:}

- Convenient online submission

- Thorough peer review

- No space constraints or color figure charges

- Immediate publication on acceptance

- Inclusion in PubMed, CAS, Scopus and Google Scholar

- Research which is freely available for redistribution 